\title{
Three-Dimensional Structures of PDE4D in Complex with Roliprams and Implication on Inhibitor Selectivity
}

Qing Huai, Huanchen Wang, Yingjie Sun, Hwa-Young Kim, Yudong Liu, and Hengming Ke* Department of Biochemistry and Biophysics and Lineberger Comprehensive Cancer Center The University of North Carolina, Chapel Hill Chapel Hill, North Carolina 27599

\section{Summary}

Selective inhibitors against the $\mathbf{1 1}$ families of cyclic nucleotide phosphodiesterases (PDEs) are used to treat various human diseases. How the inhibitors selectively bind the conserved PDE catalytic domains is unknown. The crystal structures of the PDE4D2 catalytic domain in complex with (R)- or (R,S)-rolipram suggest that inhibitor selectivity is determined by the chemical nature of amino acids and subtle conformational changes of the binding pockets. The conformational states of GIn369 in PDE4D2 may play a key role in inhibitor recognition. The corresponding Y329S mutation in PDE7 may lead to loss of the hydrogen bonds between rolipram and GIn369 and is thus a possible reason explaining PDE7's insensitivity to rolipram inhibition. Docking of the PDE5 inhibitor sildenafil into the PDE4 catalytic pocket further helps understand inhibitor selectivity.

\section{Introduction}

Cyclic nucleotide phosphodiesterase (PDE) hydrolyzes adenosine or guanosine $3^{\prime}, 5^{\prime}$-cyclic monophosphate (cAMP or cGMP) to $5^{\prime}$-AMP or 5'-GMP (Torphy, 1998; Conti and Jin, 1999; Soderling and Beavo, 2000). Cyclic AMP and cyclic GMP are intracellular second messengers, mediating the response of cells to a wide variety of hormones and neurotransmitters in signal transduction pathways. Regulation of CAMP and cGMP concentration in vivo is an essential step for many metabolic processes, such as cardiac and smooth muscle contraction, glycogenolysis, platelet aggregation, secretion, lipolysis, learning, ion channel conductance, apoptosis, and growth control (Houslay and Milligan, 1997; Houslay, 1998; Antoni, 2000).

To date, 11 families and 21 genes of PDEs have been reported (Torphy, 1998. Houslay and Milligan, 1997; Corbin and Francis, 1999; Houslay et al., 1998; Manganiello et al., 1995; Mehats et al., 2002; Müller et al., 1996; Thompson, 1991; Zhao et al., 1997). The mRNAs of the PDE genes are subject to alternative splicing, resulting in over 60 PDE isoforms in various human tissues. The molecules of all PDE families contain a conserved catalytic domain of about 300 amino acids with an approximate $25 \%$ sequence homology. However, each PDE family recognizes a specific substrate and possesses its own selective inhibitors. Thus, the families of PDE4,

*Correspondence: hke@med.unc.edu
7 , and 8 prefer to hydrolyze cAMP, while PDE5, 6 , and 9 are cGMP specific. PDE1, 2, 3, 10, and 11 take both CAMP and CGMP as their substrates. In the past three decades, selective inhibitors against the different PDE families have been widely studied as cardiotonic agents, vasodilators, smooth muscle relaxants, antidepressants, antithrombotic compounds, antiasthma compounds, and agents for improving cognitive functions such as memory (Reilly and Mohler, 2001; Rotella, 2002; Giembycz, 2000; Souness et al., 2000; Huang et al., 2001). For example, the PDE5 inhibitor sildenafil (Viagra; Figure 1) is a drug for male erectile dysfunction, and the PDE3 inhibitor cilostamide is a drug for heart diseases. Selective inhibitors of PDE4 form the largest group of inhibitors for any PDE family and have been studied as anti-inflammatory drugs targeting asthma and chronic obstructive pulmonary disease (COPD) and also as therapeutic agents for rheumatoid arthritis, multiple sclerosis, type II diabetes, septic shock, atopic dermatitis, and other autoimmune diseases (Giembycz, 2000; 2002; Souness et al., 2000; Huang et al., 2001; Piaz and Giovannoni, 2000; Barnette and Underwood, 2000; Sturton and Fitzgerald, 2002). The PDE4 selective inhibitor rolipram (Figure 1) was studied as an antidepressant and has been used to classify the PDE families (Bobon et al., 1988; Scott et al., 1991). How selective inhibitors with different chemical structures (Figure 1) bind to the similar catalytic pockets of PDEs is unknown. Herein, we report the crystal structures of the catalytic domain of human PDE4D2 (the second splicing species in the PDE4D subfamily) in complex with (R)- or (R,S)-rolipram (Table 1). These structures reveal the selective binding of roliprams to PDE4. The sequence alignment shows a conserved variation of the rolipram binding residues across the PDE families, suggesting that the inhibitor selectivity is determined by the chemical nature of amino acids and the conformational variation of the binding pockets.

\section{Results and Discussion}

Architecture of the PDE4D-Rolipram Structure Four molecules of the catalytic domain of PDE4D2 with amino acids 79-438 are associated into a tetramer in the crystallographic asymmetric unit (Figure 2). The monomeric PDE4D2 molecule contains 16 helices and has the same folding as PDE4B, except for the random loop of residues 422-434 in PDE4D2 that corresponds to helix H17 in PDE4B (Xu et al., 2000). A superposition of the catalytic domain of (R)-rolipram-bound PDE4D2 over unligated PDE4B shows an rms deviation of $0.57 \AA$ for $\mathrm{C} \alpha$ atoms, indicating the structural similarity between the PDE4 subfamilies and also implying no dramatic overall conformational changes upon binding of (R)-rolipram (Figure 3). However, substantial changes are observed for several loops. The most significant movement is the Met357 loop, which showed 2-3 times the average deviation. Since Met357 forms van der Waals interac- 


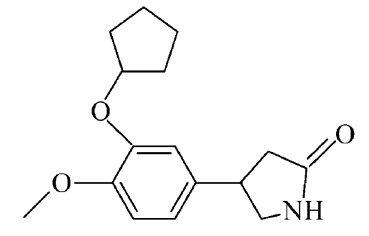

Rolipram<smiles>COc1ccc(C2(C=N)CCC(C(=O)O)CC2)cc1OC1CCCC1</smiles>

Cilomilast

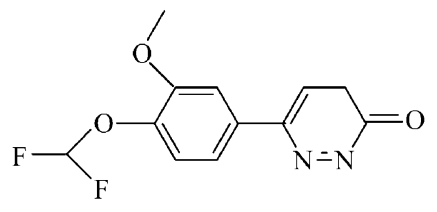

Zardavarine

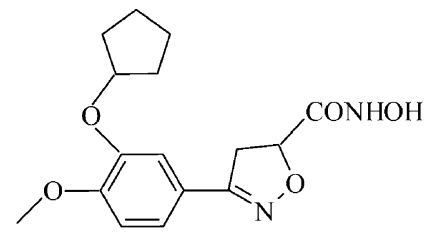

CP-293,121
Figure 1. Chemical Structures of the PDE Inhibitors

Rolipram, cilomilast, and CP-293,121 are PDE4 selective inhibitors. Zardavarine is a dual inhibitor of PDE3 and PDE4. Cilostamide is a PDE3 selective inhibitor. Sildenafil is a PDE5 selective inhibitor.<smiles>CN(C(=O)CCCOc1ccc2[nH]c(=O)ccc2c1)C1CCCCC1</smiles>

Cilostamide<smiles>CCCc1nn(C)c2c(=O)[nH]c(-c3cc(S(=O)(=O)N4CCN(C)CC4)ccc3OCC)nc12</smiles>

Sildenafil tions with (R)- and (S)-roliprams, the conformational change of this loop is likely the consequence of rolipram binding.

In spite of the tetrameric form in our crystals, a PDE4D dimer was proposed as the active catalytic form of the enzyme on the basis of the crystal structure of the PDE4D catalytic domain in complex with zardavarine (Lee et al., 2002). This argument is supported by the fact that a dimer is observed in a different crystal form of PDE4D2 that was grown in the presence of $0.1 \mathrm{M}$ calcium and 1 $\mathrm{mM}(\mathrm{R})$-rolipram (our unpublished data) and that the molecular sieving column in the protein purification showed a peak with an apparent molecular mass of 75 $\mathrm{kDa}$ that is equivalent to a dimer of the catalytic domain of PDE4D2. However, the PDE4B domain with amino acids $152-528$ that retains catalytic activity is a monomer in crystals (Xu et al., 2000; Rocque et al., 1997), and the PDE4A catalytic domain in the various oligomerization states from monomer to tetramer is active (Lario et al., 2001). Thus, the crystallographic and biochemical studies suggest that either monomer, dimer, or tetramer may be catalytically active, depending on the isoforms of the enzyme.

\section{Rolipram Binding}

The (R)- and (R,S)-roliprams bind to the catalytic domain of the recombinant PDE4D2 with $\mathrm{IC}_{50}$ values of 0.33 and
$0.55 \mu \mathrm{M}$, respectively (Dr. Wei Zhang, personal communication). The crystal structures showed that the (R)and $(\mathrm{S})$-enantiomers of rolipram bind to the active site of PDE4D2 with similar orientations and interact with the same residues (Figure 4). Roliprams form two hydrogen bonds with the side chain of GIn369 and numerous hydrophobic interactions with the active site residues. The cyclopentyloxy groups of (R)- and (S)-roliprams sit in a hydrophobic pocket, interacting with residues Ile336, Met337, Phe340, Met357, Ser368, GIn369, and Phe372. The phenylmethoxy rings of (R)- and (S)-roliprams stack over Phe372 and also contact Tyr159, Asn321, Tyr329, Thr333, Ile336, and Gln369 via van der Waals forces. Although the pyrrolidone groups of (R)- and (S)-roliprams have an opposite chirality, they unexpectedly interact with the same residues of PDE4D2. Surprisingly, the pyrrolidone rings, which are anchored in the direction of the metal binding pocket, form no hydrogen bonds with the active site residues or divalent metals, but form polar interactions with His160, Met273, Leu319, Phe340, and Phe372. The interactions of (R)- and (S)-roliprams with the active site residues in the crystal structure agree qualitatively with, but are somewhat different in detail from, the prediction of rolipram binding in the docked model (Dym et al., 2002). Comparison of (R)-rolipram binding with the docked model of cAMP (Xu et al., 2000) shows overlap of the phenylmethoxy 


\begin{tabular}{|c|c|c|}
\hline \multicolumn{3}{|l|}{ Data Collection } \\
\hline & (R,S)-Rolipram & (R)-Rolipram \\
\hline Space group & $\mathrm{P} 2{ }_{1} 2_{1} 2_{1}$ & $\mathrm{P} 2{ }_{1} 2_{1} 2_{1}$ \\
\hline Unit cell $(a, b, c)(\AA)$ & $99.8,111.5,160.0$ & $99.3,112.5,160.9$ \\
\hline Resolution (Å) & 2.0 & 2.3 \\
\hline Total measurements & 677,028 & 491,376 \\
\hline Unique reflections & 89,779 & 77,976 \\
\hline Completeness (\%) & $86.7(69.1)^{a}$ & $97.0(75.6)$ \\
\hline Average $\mathrm{I} / \sigma$ & $20.0(3.8)$ & $14.0(2.8)$ \\
\hline $\mathbf{R}_{\text {merge }}$ & $0.065(0.54)$ & $0.053(0.246)$ \\
\hline \multicolumn{3}{|l|}{ Structure Refinement } \\
\hline $\mathrm{R}$ factor & 0.233 & 0.224 \\
\hline $\mathbf{R}_{\text {free }}$ & 0.266 (7.4\%) & $0.260(9.5 \%)$ \\
\hline Resolution & $50-2.0 \AA$ & $50-2.3$ \\
\hline Reflections & 89,775 & 75,736 \\
\hline \multicolumn{3}{|l|}{ RMS deviation } \\
\hline Bond $(\AA)$ & 0.0061 & 0.0062 \\
\hline Angle $\left({ }^{\circ}\right)$ & 1.21 & 1.19 \\
\hline \multicolumn{3}{|l|}{ Average $\mathrm{B}$ factor $\left(\AA^{2}\right)$} \\
\hline All atoms & 41.8 & 38.5 \\
\hline Protein & 41.9 & 38.7 \\
\hline Inhibitor & 48.8 & 37.2 \\
\hline Zn1 & 35.0 & 32.3 \\
\hline $\mathrm{Zn} 2$ & 62.0 & 62.6 \\
\hline Water & 32.7 & 27.1 \\
\hline
\end{tabular}

group of (R)-rolipram over adenosine of cAMP. This is consistent with the earlier prediction of Kleinman et al. (1998) on the basis of structure-activity relationships of the hydroxamate-containing inhibitor CP-293121.

The small contribution of the pyrrolidone ring to rolipram binding as observed in our crystal structure predicts a similar affinity of (R)- and (S)-roliprams to the PDE4 family. However, the results from the biochemical study are controversial. One group reported that (R)and (S)-roliprams show no observable differences in binding affinity and inhibition of cAMP hydrolysis between the four subfamilies of PDE4 (Demnitz et al., 1998). In contrast, the results from other groups showed that (R)-rolipram has 10- to 20-fold tighter binding and 3- to 10-fold more inhibition of cAMP hydrolysis than (S)-rolipram (Torphy et al., 1992; Barnette et al., 1996; Laliberte et al., 2000). The reason for the differing results is not clear. One possibility might be the fluctuation of the molecular conformation of PDE4 in solution, which may depend on protein preparation under certain chemical environments, such as metal ion concentration in solution. The metal dependence of rolipram affinity was reported, as shown by the case that the 12-fold stereoselectivity of (R)-rolipram over (S)-rolipram in the holoenzyme of PDE4A (the enzyme with the divalent metals) was reduced to about 2-fold in the apoenzyme (the enzyme without metals; Liu et al., 2001). Our preliminary study on the different crystal form grown in the presence of $0.1 \mathrm{M} \mathrm{CaCl}_{2}$ showed an extra peak, which was tentatively interpreted as a calcium ion and interacted with the two metals and pyrrolidone (our unpublished data). Thus, the additional interactions of the peak with pyrrolidone might account for the high affinity of rolipram in the presence of metal (Liu et al., 2001).
Recently, the crystal structure of the catalytic domain of PDE4D in complex with the inhibitor zardavarine has been reported (Lee et al., 2002). Zardavarine binds to the same hydrophobic pocket as rolipram and interacts with residues Tyr159, Met273, Leu319, Ile336, Met337, Phe340, Met357, Gln369, and Phe372 (PDE4D2 numbering). It forms hydrogen bonds with Gln369 and Tyr159 but does not directly interact with the metals. The crystal structures of PDE4D in complex with rolipram and zardavarine suggest that all the PDE4 inhibitors, whether they have similar or different chemical structures, would share a similar set of binding residues, although the detailed interactions or partnership may vary from inhibitor to inhibitor. Since zardavarine is a dual inhibitor of PDE3 and PDE4 (Underwood et al., 1994), the information on the binding pocket as revealed in the PDE4Dinhibitor structures might be useful for the design of inhibitors against other PDE families.

\section{Implications on Inhibitor Selectivity}

The sequence homology suggests that the PDE families share a similar three-dimensional structure in their catalytic domains. However, each of the PDE families possesses its own selective inhibitors. Thus, the questions about inhibitor selectivity remain puzzling. How do the selective inhibitors for the different PDE families preferably bind one family of catalytic pockets among many of apparently similar shapes and sizes? How do PDE4 inhibitors with different chemical structures bind to the same pocket? What are the determinants for PDE inhibitor selectivity? The structure of PDE4D2 complexed with rolipram provides insight into the selective binding of PDE4 inhibitors and also sheds light on the inhibitor selectivity among the PDE families. 
A

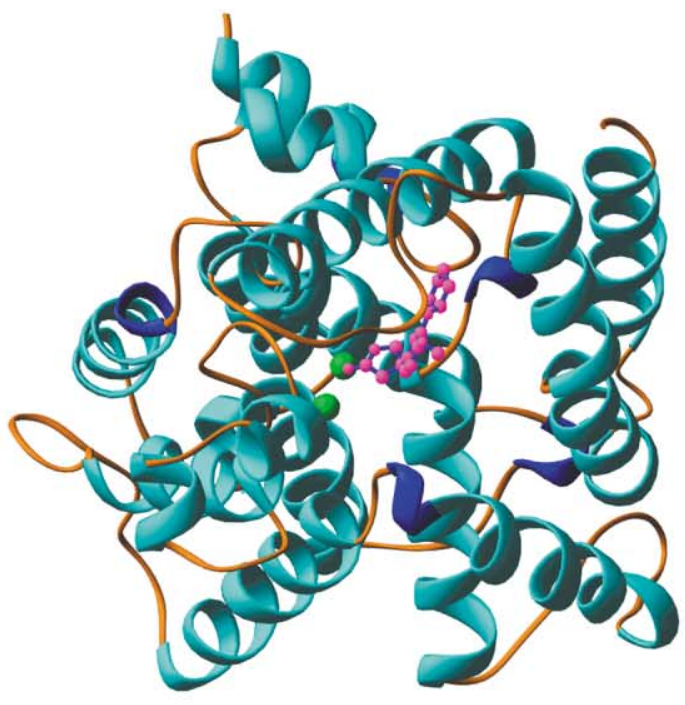

B

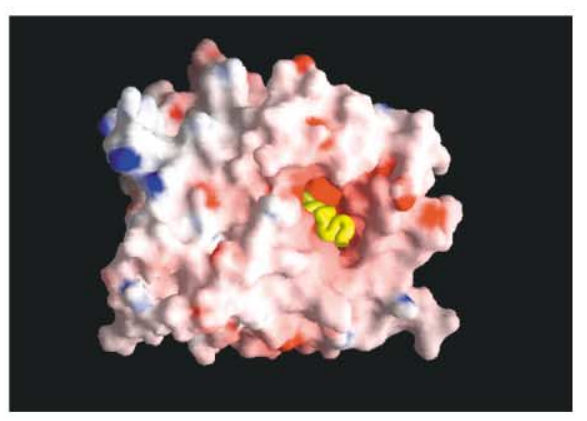

C

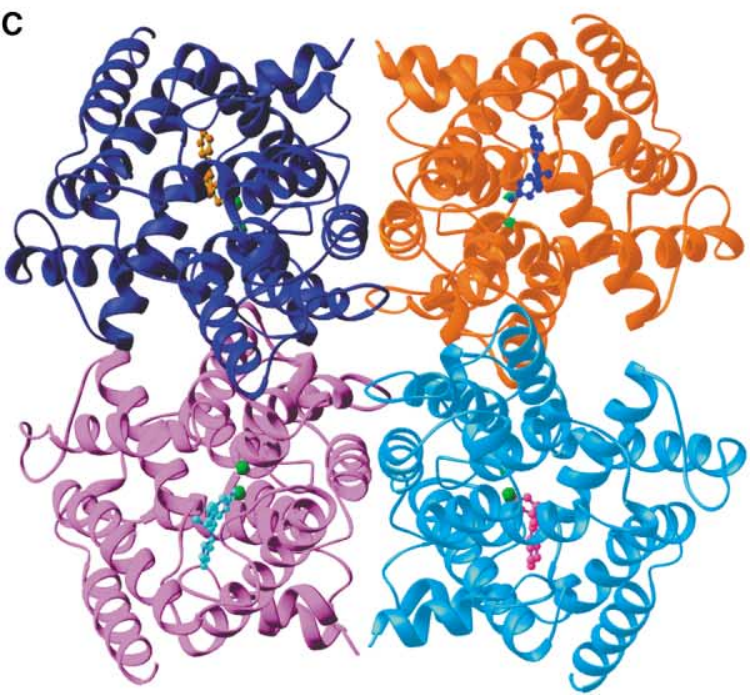

Figure 2. The Catalytic Domain of PDE4D2

(A) Ribbon diagram of the monomeric PDE4D2 catalytic domain. Rolipram, pink balls; two divalent metals, green balls.

(B) Surface presentation of the monomeric PDE4D2 catalytic domain. Rolipram, yellow noodle.

(C) Ribbons presentation of the tetramer of the PDE4D2 catalytic domain.
In terms of the geometric shape, the active sites of the PDE families can be further divided into four subpockets, respectively, for binding of divalent metals and three groups (cyclopentyloxy, phenylmethoxy, and pyrrolidone) of rolipram. The metal binding subpocket includes four metal binding residues (His164, His200, Asp201, and Asp318) and His160, which is presumably a key residue for the cAMP hydrolysis. These five residues are identical across the PDE families (Figure 4), implying a common mechanism of the binuclear catalysis for all PDEs. The subpocket for the cyclopentyloxy group of rolipram is made up of characteristic hydrophobic residues Met337, Phe340, and Met357, and may therefore accommodate a hydrophobic substitution group with a size comparable to cyclopentane. The subpocket for the phenylmethoxy group of rolipram has a "ditch"-like shape, with lle336 and Phe372 sitting on each side. Thus, for the design of new PDE4 inhibitors, modification of the phenyl group of rolipram may be limited to a planar ring to fit the ditch. In addition, the methoxy of the phenylmethoxy group occupies an opening space made up of Asn321, Tyr329, Trp332, Thr333, and GIn369. This opening space is apparently bigger than the methoxy group and therefore could accept a larger group containing either polar or nonpolar atoms. In fact, the difluoromethoxy group of zardavarine occupies the pocket and forms hydrogen bonds with GIn369 (Lee et al., 2002). The subpocket for the pyrrolidone group of rolipram is adjacent to the metal pocket and also open to the surface of the molecule. Thus, dramatic modifications on pyrrolidone could be performed to make interactions with either metals or the residues near the active site for tighter binding and better selectivity against different families of PDEs. Since the cyclopentyloxy and phenylmethoxy groups of cilomilast and CP293121 are common to rolipram (Figure 1), the binding of these groups is expected to be the same as that of rolipram. The remaining groups of cilomilast and CP293121 are expected to occupy the pyrrolidone subpocket of rolipram and to extend their interactions with the metal ions or nearby residues, thus accounting for their high affinity for PDE4.

In addition, the structure-based sequence alignment of the rolipram binding residues provides insight into inhibitor selectivity (Figure 4). GIn369 is the only rolipram binding residue absolutely conserved in all the PDE families. Since hydrogen bonds are major factors for determining the accurate orientation of a ligand, GIn369 and its conformation must play a key role in inhibitor binding and selectivity. Hydrophobic residues Tyr159, Met273, Leu319, Trp332, Ile336, Phe340, Met357, and Phe372 are conservatively substituted in other families of PDE (Figure 4). These residues contribute the hydrophobic interactions to the binding of inhibitors, and their important roles are confirmed by the mutagenesis data (Richter et al., 2001; Atienza et al., 1999). The variation of these residues will determine the size and shape of the binding pockets in different PDE families, thus defining inhibitor selectivity. Asn321, Tyr329, and Ser368 are three residues showing the largest variation across the PDE families (Figure 4) and must play critical roles in defining inhibitor selectivity. In short, we speculate that the inhibitor selectivity must be determined 

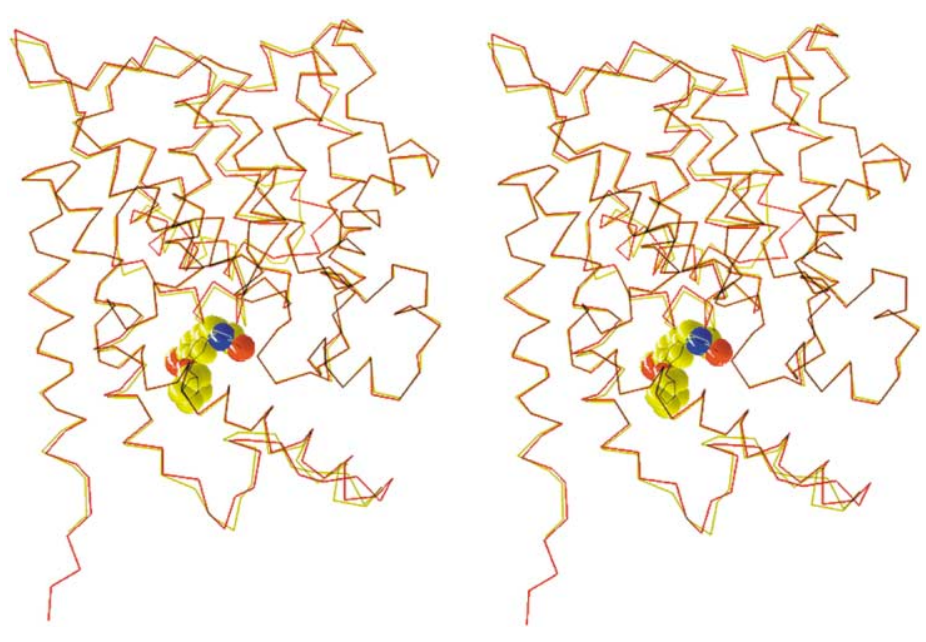

Figure 3. Stereo View of Superposition between the Catalytic Domains of PDE4D (Yellow) and PDE4B (Red)

(R)-Rolipram is shown in CPK model. by a combination of different amino acids and subtle conformational changes at the active site of each PDE family.

\section{Docking of Sildenafil into the Active Site of PDE4D} To investigate inhibitor selectivity, we modeled the PDE5 selective inhibitor sildenafil (Figure 1), a drug for treatment of male erectile dysfunction, into the catalytic pocket of PDE4D2 (Figure 5). Sildenafil selectively binds PDE5 with an $\mathrm{IC}_{50}$ value of about $3.5 \mathrm{nM}$, in comparison with an $\mathrm{IC}_{50}$ of $7.7 \mu \mathrm{M}$ for the binding to PDE4 (Corbin and Francis, 2002). The model shows an overall fit of sildenafil into the catalytic pocket of PDE4. The purinelike ring of sildenafil sits in the hydrophobic pocket and stacks against Phe372, whereas GIn369 forms a hydrogen bond with the oxygen of the purine-like ring. In addition, two oxygen atoms of the sulfonamide group of sildenafil directly contact the divalent metals via chargecharge interactions. However, the side chain of Asn321 shows a distance as short as $2 \AA$ to several atoms of the purine-like ring in the model. Thus, the native amino acid alanine in PDE5, which corresponds to Asn321, is expected to avoid the collisions and to favor hydrophobic interactions with the purine-like ring of sildenafil. Since Asn321 dramatically varies across the PDE families (Figure 4), a critical role for this residue in inhibitor selectivity is proposed. This argument is supported by the results that the same Asn to Ala replacement in PDE6 and PDE11 also shows the high sildenafil selectivity (Corbin and Francis, 2002).

\section{Insensitivity of PDE7 to Rolipram Inhibition}

Like PDE4, PDE7 is highly cAMP specific, but rolipram insensitive. Thus, to further investigate inhibitor selectivity, we modeled the catalytic pocket of PDE7. Sequence alignment of PDE4 and PDE7 showed that 6 out of 14 rolipram binding residues are identical between the two families, whereas the remaining 8 residues are conservatively mutated (Figure 4). A careful examination of the PDE4D-rolipram structure suggests that the replacement of corresponding Tyr329 with serine in PDE7 might be the key to switch the highly specific binding of rolipram in PDE4 to no binding in PDE7. The hydrogen bond of Tyr329 with GIn369, which is the only residue absolutely conserved across PDE families, may serve as a scaffold to support formation of the hydrogen bonds between Gln369 and rolipram (Figure 6). We speculate that the hydrogen bonding network of Tyr329-GIn369rolipram must play a key role in the recognition of the inhibitors. The potential loss of the hydrogen bond between Tyr329 and GIn369 by the mutation of tyrosine to serine in PDE7 is thus expected to dramatically change the side chain conformation of GIn369 in order to form a hydrogen bond with Ser373 in PDE7 (Figure 6). As a result, the hydrogen bonds between rolipram and GIn369 in PDE4 may be absent in PDE7, to diminish the rolipram inhibition in PDE7.

\section{Biological Implications}

Eleven families of human PDEs in different human tissues are involved in regulation of cAMP and cGMP concentrations in various biological processes. Each family of PDEs possesses its own selective inhibitors that bind to the conserved catalytic domains. It has remained a mystery how the inhibitors with different structures selectively inhibit the similar catalytic pocket of PDEs. The crystal structures of PDE4D2 in complex with (R)or (R,S)-rolipram reveal the detailed binding of the inhibitors. The sequence alignment of the rolipram binding residues shows that the majority of the residues are well conserved. Among them, Gln369 is the only identical residue in the PDE families and forms hydrogen bonds with rolipram. Therefore, the conformational state of Gln369 will be critical for binding of the inhibitors and differentiating inhibitor selectivity. Since PDE7 contains serine in the corresponding position of Tyr329, Gln369 might loose the capability of forming hydrogen bonds with rolipram because of the possible loss of the supporting hydrogen bond between Tyr329 and GIn369. This may be a reason to account for the insensitivity of PDE7 to rolipram inhibition. Asn321 is a residue that changes dramatically across the PDE families and may play a critical role in the recognition of inhibitors. To investigate inhibitor selectivity, we docked the PDE5 selective inhibitor silnadefil into the active site of PDE4D2. Overall, sildenafil fits the pocket, but several atoms of the purine-like ring show a distance as short as $2 \AA$ to the side chain of Asn321. Correspondence 
A
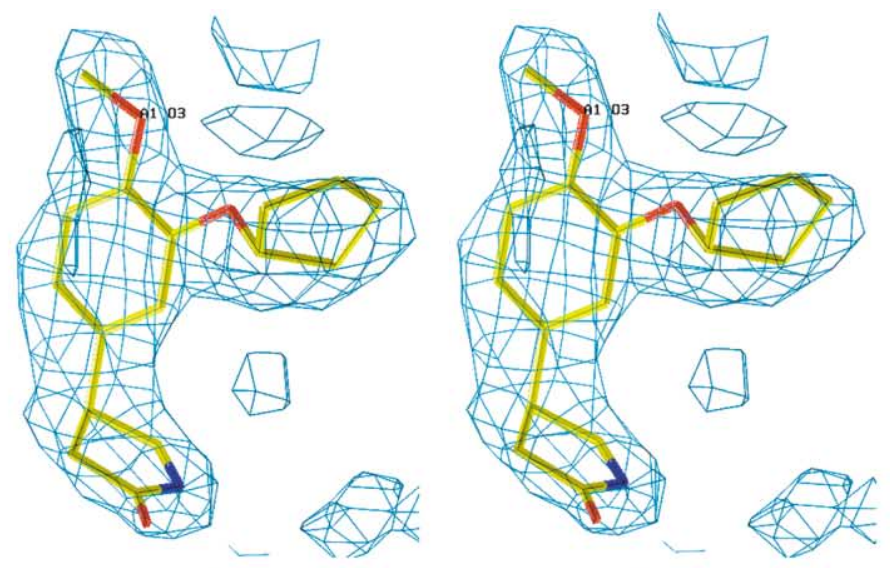

B
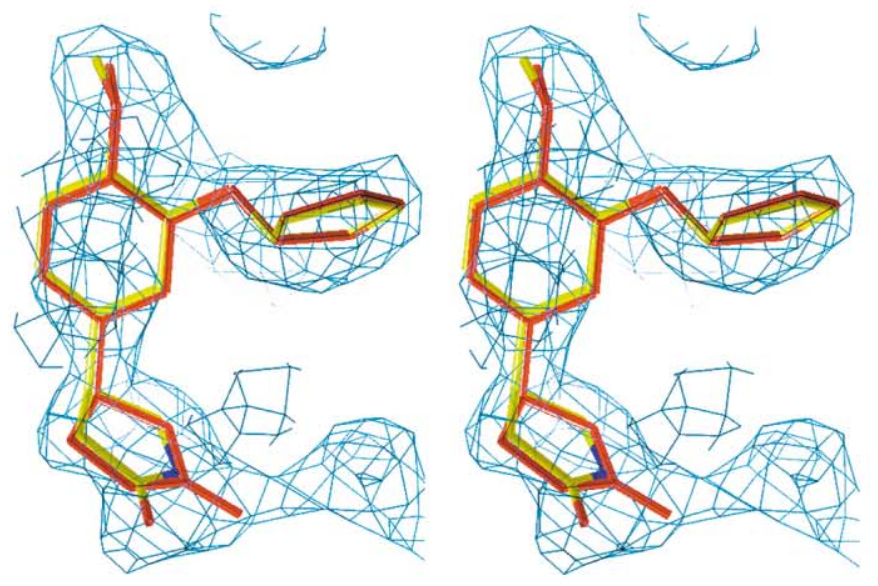

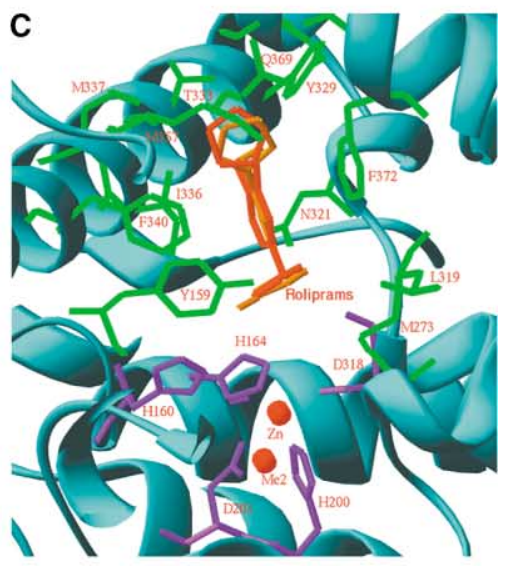

D

$\begin{array}{lllllllllllll}160 & 164 & 200 & 273 & 318 & 321 & 329 & 332 & 336 & 340 & 357 & 368 & 372\end{array}$

$\begin{array}{llllllllllllll}\text { pde4d2 } & \text { YH } & \text { H } & \text { HD } & \text { M } & \text { DL } & \text { N } & \text { Y } & \text { WT } & \text { IM } & \text { F } & \text { M } & \text { SQ } & \text { F } \\ \text { pde4b2b } & \text { YH } & \text { H } & \text { HD } & \text { M } & \text { DL } & \text { N } & \text { Y } & \text { WT } & \text { IM } & \text { F } & \text { M } & \text { SQ } & \text { F } \\ \text { pde3al } & \text { YH } & \text { H } & \text { HD } & \text { L } & \text { DI } & \text { G } & \text { H } & \text { WT } & \text { IV } & \text { F } & \text { F } & \text { LQ } & \text { F } \\ \text { pde7a } & \text { YH } & \text { H } & \text { HD } & \text { I } & \text { DI } & \text { N } & \text { S } & \text { WS } & \text { VT } & \text { F } & \text { L } & \text { IQ } & \text { F } \\ \text { pde8a } & \text { YH } & \text { H } & \text { HD } & \text { M } & \text { DV } & \text { N } & \text { C } & \text { WA } & \text { IS } & \text { Y } & \text { V } & \text { SQ } & \text { F } \\ \text { pde1a3a } & \text { YH } & \text { H } & \text { HD } & \text { M } & \text { DI } & \text { H } & \text { H } & \text { WT } & \text { LM } & \text { F } & \text { L } & \text { SQ } & \text { F } \\ \text { pde2a3 } & \text { YH } & \text { H } & \text { HD } & \text { L } & \text { DL } & \text { D } & \text { T } & \text { IA } & \text { IY } & \text { F } & \text { M } & \text { LQ } & \text { F } \\ \text { pde10a2 } & \text { YH } & \text { H } & \text { HD } & \text { L } & \text { DL } & \text { S } & \text { T } & \text { TA } & \text { IY } & \text { F } & \text { M } & \text { GQ } & \text { F } \\ \text { pde11a } & \text { YH } & \text { H } & \text { HD } & \text { L } & \text { DL } & \text { A } & \text { S } & \text { VA } & \text { VT } & \text { F } & \text { I } & \text { LQ } & \text { W } \\ \text { pde5a1 } & \text { YH } & \text { H } & \text { HD } & \text { L } & \text { DL } & \text { A } & \text { Q } & \text { IA } & \text { VA } & \text { F } & \text { L } & \text { MQ } & \text { F } \\ \text { pde6a } & \text { YH } & \text { H } & \text { HD } & \text { L } & \text { DL } & \text { A } & \text { Q } & \text { VA } & \text { VA } & \text { F } & \text { M } & \text { LQ } & \text { F } \\ \text { pde9aI } & \text { FH } & \text { H } & \text { HD } & \text { M } & \text { DI } & \text { N } & \text { A } & \text { WV } & \text { LI } & \text { Y } & \text { F } & \text { AQ } & \text { F }\end{array}$

Figure 4. Binding of (R)- and (S)-Roliprams

(A) Stereo view of the electron density for (R)-rolipram. The omitted $\left(2 F_{\circ}-F_{c}\right)$ map was contoured at $1.5 \sigma$.

(B) Stereo view of the electron density for (R,S)-rolipram. (R)-rolipram, yellow sticks; (S)-rolipram, orange.

(C) Rolipram binding at the catalytic pocket of PDE4D2. Zinc coordinates with His164, His200, Asp201, and Asp318 (purple). The second metal (Me2) is tentatively interpreted as magnesium and binds to Asp318 and a water molecule. (R)- and (S)-roliprams bind to the hydrophobic pocket in very similar orientations and interact with the same residues of PDE4. They form hydrogen bonds only with Gln369 but do not directly interact with the divalent metals.

(D) Sequence alignment of the rolipram binding residues between 11 PDE families. The metal binding residues are marked with asterisks. Most of the rolipram binding residues are conserved, but not identically, implying their key roles in defining of inhibitor selectivity. 


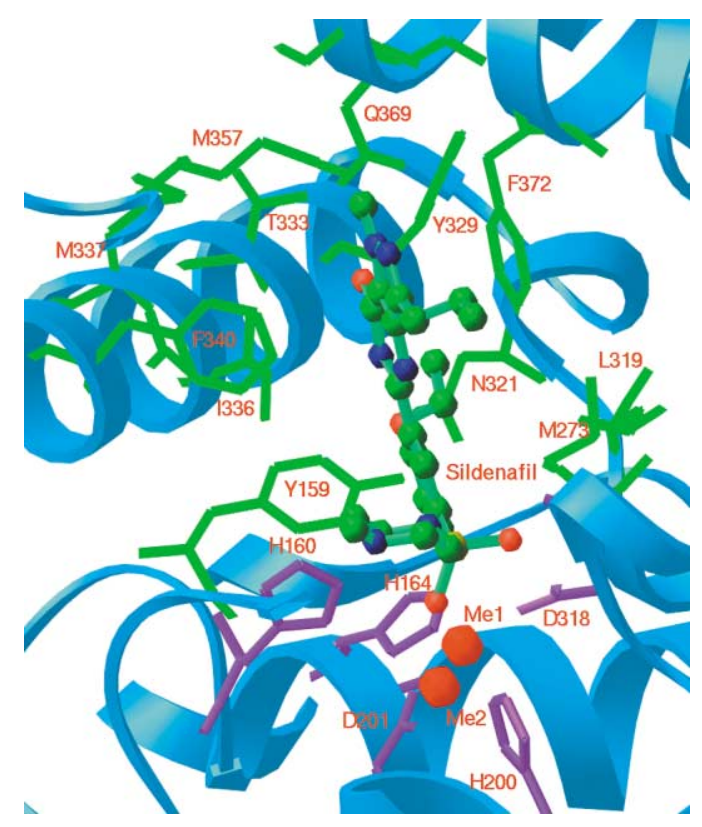

Figure 5. Model of PDE5 Selective Inhibitor Sildenafil (Viagra) Bound in the Catalytic Pocket of PDE4D2

Overall, sildenafil fits in the pocket, but several atoms of the purinelike ring of sildenafil are located at a distance as short as $2 \AA$ from the side chain of Asn321. The correspondence of alanine to Asn321 in PDE5, 6, and 11 is expected to favor hydrophobic interactions, thus possibly explaining their high selectivity.

of alanine to Asn321 in PDE5, 6, and 11 might favor hydrophobic interactions between the inhibitor and the proteins, thus explaining the high sildenafil selectivity of these proteins. In summary, our work suggests that inhibitor selectivity is defined by both the chemical nature of amino acids and the conformational variation of the binding pockets.

\section{Experiment Procedures}

\section{Protein Expression and Purification}

The EST (expressed sequence tag) cDNA clones of PDE4D2 (BF059733) were purchased from ATCC and subcloned by standard methods. The coding regions for amino acids 79-438 of PDE4D2 were amplified by PCR and subcloned into the expression vector $\mathrm{pET} 15 \mathrm{~b}$. The resulting plasmid $\mathrm{pET}-\mathrm{PDE}$ 4D2 was transformed into $E$. coli strain BL21 (Codonplus) for overexpression. The E. coli cell carrying PET-PDE4D2 was grown in LB medium at $37^{\circ} \mathrm{C}$ to the absorption $A 600=0.7$, and then $0.1 \mathrm{mM}$ isopropyl $\beta$-D-thiogalactopyranoside was added for further growth at $12^{\circ} \mathrm{C}$ for $40 \mathrm{hr}$. The recombinant PDE4D2 was purified by Ni-NTA affinity column (Qiagen), thrombin cleavage, Q Sepharose (Phamacia), and Superdex 200 (Phamacia) columns. The PDE4D2 protein has purity greater than $95 \%$ as shown by SDS-PAGE. A typical purification yielded over 100 mg PDE4D2 from a 2 liter cell culture.

\section{Crystallization and Data Collection}

The crystals were obtained by vapor diffusion with $15 \mathrm{mg} / \mathrm{ml}$ PDE4D2 in a storage buffer of $50 \mathrm{mM} \mathrm{NaCl}, 20 \mathrm{mM}$ Tris- $\mathrm{HCl}(\mathrm{pH}$ 7.5), $1 \mathrm{mM} \beta$-mercaptoethanol, and $1 \mathrm{mM}$ EDTA. The crystals of the catalytic domain of PDE4D2 (amino acids 79-438) in complex with (R)-rolipram were grown against a well buffer of $0.1 \mathrm{M} \mathrm{HEPES}(\mathrm{pH}$ 7.5), $20 \%$ PEG $3350,30 \%$ ethylene glycol, $10 \%$ isopropanol, and $5 \%$ glycerol at $4^{\circ} \mathrm{C}$. It forms in space group $\mathrm{P} 2{ }_{1} 2_{1} 2_{1}$, with cell dimensions of $a=99.3, b=112.5$, and $c=160.9 \AA$. The diffraction data were collected on beamline X25 at the Brookhaven National
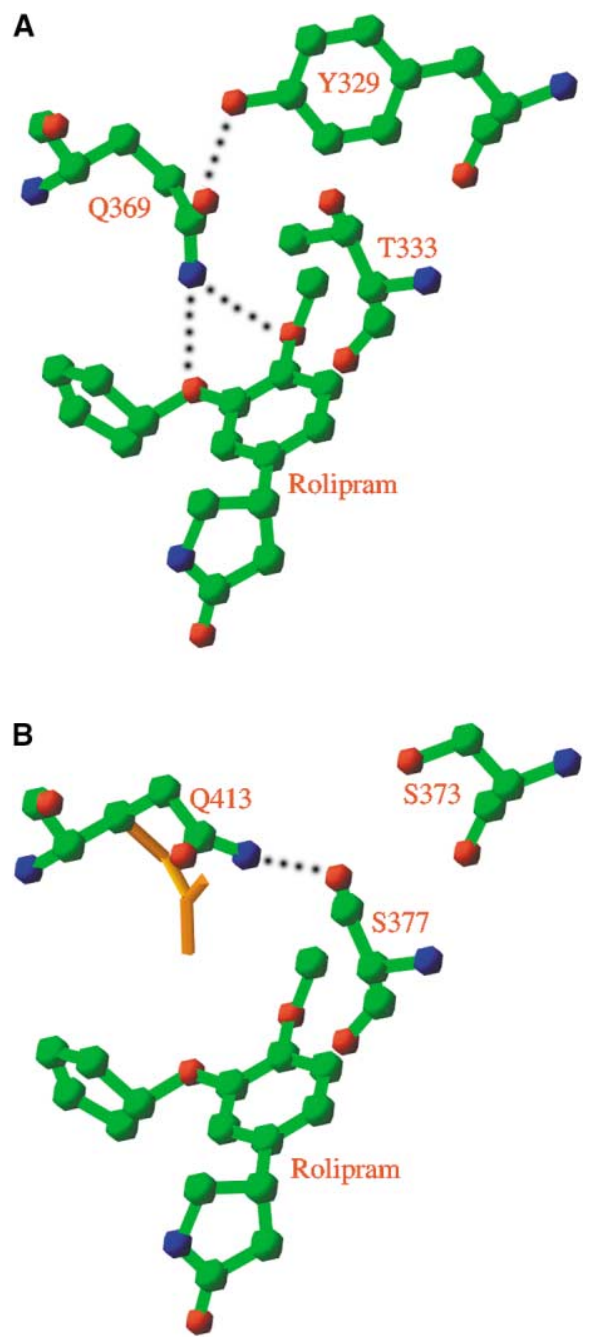

Figure 6. A Model for Insensitivity of PDE7 to Rolipram Inhibition (A) The hydrogen bonds (dotted lines) between GIn369 and rolipram in PDE4.

(B) The correspondence of residues Ser373, Ser377, and GIn413 in PDE7A to Tyr329, Thr333, and GIn369 in PDE4D2 may lead Gln413 to adopt a new conformation to form a hydrogen bond with Ser377. As a result, Gln413 would not be capable of forming hydrogen bonds with rolipram. The orange sticks mark the side chain conformation of GIn369 in PDE4.

Laboratory (Table 1). The catalytic domain of PDE4D2 (amino acids 79-438) in complex with racemic (R, S)-rolipram was crystallized against a well buffer of 0.05 M HEPES (pH 7.5), 20\% PEG 3350, $25 \%$ ethylene glycol, and $10 \%$ isopropanol at $4^{\circ} \mathrm{C}$. It forms in space group $P 22_{1} 2_{1} 2_{1}$, with cell dimensions of $\mathrm{a}=99.8, \mathrm{~b}=111.5$, and $\mathrm{c}=$ $160.0 \AA$. The diffraction data were collected on beamline $14 \mathrm{C}$ of APS at the Argonne National Laboratory (Table 1) and processed with the program HKL (Otwinowski and Minor, 1997).

\section{Structure Determination}

The crystals of PDE4D2 in complex with roliprams contain a tetramer in the crystallographic asymmetric unit. The structure of PDE4D2rolipram was solved by the molecular replacement program AMoRe (Navaza and Saludjian, 1997), with the catalytic domain of PDE4B as the initial model (Xu et al., 2000) and a preliminary $2.8 \AA$ resolution data set that was collected on beamline $\mathrm{X} 12 \mathrm{C}$ at the Brookhaven National Lab. The translation search of a monomer yielded a correlation coefficient of 0.20 and an $\mathrm{R}$ factor of 0.51 for 10,784 reflections 
between 4 and $8 \AA$ A resolution. The translational search for the remaining molecules under the fixed orientation of the first molecule produced three solutions with correlation coefficients of $0.31,0.33$, and 0.23 and $R$ factors of $0.48,0.47$, and 0.51 , respectively. The tetramer of PDE4D-rolipram was optimized by rigid-body refinement with CNS (Brünger et al., 1998). The electron density map was improved by the density modification package of CCP4 (CCP4, 1994). The atomic model was rebuilt with the program $\mathrm{O}$ (Jones et al., 1991) and refined with CNS (Table 1).

\section{Acknowledgments}

We wish to thank Beamlines X25 and X12C at the NSLS and beamline $14 \mathrm{C}$ at the APS for collection of diffraction data, Drs. E.F. Kleinman and A.J. Duplantier of Pfizer for the gift of (R)-rolipram, Drs. E. Kleinman and M. Topal for proofreading of the manuscript, and Drs. L. Betts and W. Zhang for technical support. This work was supported in part by the NIH (GM59791 to H.K).

Received: January 14, 2003

Accepted: April 2, 2003

Published: July 1, 2003

\section{References}

Antoni, F. (2000). Molecular diversity of cyclic AMP signaling. Front. Neuroendocrinol. 21, 103-132.

Atienza, J.M., Susanto, D., Huang, C., McCarty, A.S., and Colicelli, J. (1999). Identification of inhibitor specificity determinants in a mammalian phosphodiesterase. J. Biol. Chem. 274, 4839-4847.

Barnette, M.S., Bartus, J.O., Burman, M., Christensen, S.B., Cieslinski, L.B., Esser, K.M., Prabhakar, U.S., Rush, J.A., and Torphy, T.J. (1996). Association of the anti-inflammatory activity of phosphodiesterase 4 (PDE4) inhibitors with either inhibition of PDE4 catalytic activity or competition for [3H]rolipram binding. Biochem. Pharmacol. 51, 949-956.

Barnette, M.S., and Underwood, D.C. (2000). New phosphodiesterase inhibitors as therapeutics for the treatment of chronic lung disease. Curr. Opin. Pulm. Med 6, 164-169.

Bobon, D., et al. (1988). Is phosphodiesterase inhibition a new mechanism of antidepressant action? A double-dummy study between rolipram and desipramine in hospitallized major and/or endogenous depressives. Eur. Arch. Psychiatry Neurol. Sci. 238, 2-6.

Brünger, A.T., Adams, P.D., Clore, G.M., DeLano, W.L., Gros, P., Grosse-Kunstleve, R.W., Jiang, J.S., Kuszewski, J., Nilges, M., Pannu, N.S., et al. (1998). Crystallography and NMR System: a new software suite for macromolecular structure determination. Acta Crystallogr. D Biol. Crystallogr. 54, 905-921.

CCP4 (Collaborative Computational Project 4). (1994). The CCP4 suite: programs for protein crystallography. Acta Crystallogr. D Biol. Crystallogr. 50, 760-763.

Conti, M., and Jin, S.L. (1999). The molecular biology of cyclic nucleotide phosphodiesterases. Prog. Nucleic Acid Res. Mol. Biol. 63, 1-38.

Corbin, J.D., and Francis, S.H. (1999). Cyclic GMP phosphodiesterase-5: target of sildenafil. J. Biol. Chem. 274, 13729-13732.

Corbin, J.D., and Francis, S.H. (2002). Pharmacology of phosphodiesterase-5 inhibitors. Int. J. Clin. Pract. 56, 453-459.

Demnitz, J., LaVecchia, L., Bacher, E., Keller, T.H., Müller, T., Schürch, F., Weber, H.P., and Pombo-Villar, E. (1998). Enantiodivergent synthesis of (R)- and (S)-rolipram. Molecules 3, 107-119.

Dym, O., Xenarios, I., Ke, H., and Colicelli, J. (2002). Molecular docking of competitive phosphodiesterase inhibitors. Mol. Pharmacol. 61, 20-25.

Giembycz, M.A. (2000). Phosphodiesterase 4 inhibitors and the treatment of asthma. Drugs 59, 193-212.

Giembycz, M.A. (2002). Development status of second generation PDE 4 inhibitors for asthma and COPD: the story so far. Monaldi Arch. Chest Dis. 57, 48-64.

Houslay, M.D. (1998). Adaptation in cyclic AMP signaling processes: a central role for cyclic AMP phosphodiesterases. Semin. Cell Dev. Biol. 9, 161-167.

Houslay, M.D., and Milligan, G. (1997). Tailoring cAMP-signalling responses through isoform multiplicity. Trends Biochem. Sci. 22, 217-224.

Houslay, M.D., Sullivan, M., and Bolger, G.B. (1998). The multienzyme PDE4 cyclic adenosine monophosphate-specific phosphodiesterase family: intracellular targeting, regulation, and selective inhibition by compounds exerting anti-inflammatory and antidepressant actions. Adv. Pharmocol. 44, 225-343.

Huang, Z., Ducharme, Y., Macdonald, D., and Robichaud, A. (2001). The next generation of PDE4 inhibitors. Curr. Opin. Chem. Biol. 5, 432-438.

Jones, T.A., Zou, J.-Y., Cowan, S.W., and Kjeldgaard, M. (1991). Improved methods for building protein models in electron density maps and the location of errors in these models. Acta Crystallogr. A 47, 110-119.

Kleinman, E.F., Campbell, E., Giordano, L.A., Cohan, V.L., Jenkinson, T.H., Cheng, J.B., Shirley, J.T., Pettipher, E.R., Salter, E.D., Hibbs, T.A., et al. (1998). Striking effect of hydroxamic acid substitution on the phosphodiesterase type 4 (PDE4) and TNF inhibitory activity of two series of rolipram analogues: implications for a new active site model of PDE4. J. Med. Chem. 41, 266-270.

Laliberte, F., Han, Y., Govindarajan, A., Giroux, A., Liu, S., Bobechko, B., Lario, P., Bartlett, A., Gorseth, E., Gresser, M., et al. (2000). Conformational difference between PDE4 apoenzyme and holoenzyme. Biochemistry 39, 6449-6458.

Lario, P.I., Bobechko, B., Bateman, K., Kelly, J., Vrielink, A., and Huang, Z. (2001). Purification and characterization of the human PDE4A catalytic domain (PDE4A330-723) expressed in Sf9 cells. Arch. Biochem. Biophys. 394, 54-60.

Lee, M.E., Markowitz, J., Lee, J.O., and Lee, H. (2002). Crystal structure of phophodiesterase 4D and inhibitor complex. FEBS Lett. 530, 53-58.

Liu, S., Laliberte, F., Bobechko, B., Bartlett, A., Lario, P., Gorseth, E., Van Hamme, J., Gresser, M.J., and Huang, Z. (2001). Dissecting the cofactor-dependent and independent binding of PDE4 inhibitors. Biochemistry 40, 10179-10186.

Manganiello, V.C., Taira, M., Degerman, F., and Belfrage, P. (1995). Type III cGMP-inhibited cyclic nucleotide phosphodiesterase (PDE3 gene family). Cell. Signal. 7, 445-455.

Mehats, C.M., Anderson, C.B., Filopanti, M., Jin, S.L.C., and Conti, M. (2002). Cyclic nucleotide phosphodiesterases and their role in endocrine cell signaling. Trends Endocrinol. Metab. 13, 29-35.

Müller, T., Engels, P., and Fozard, J.R. (1996). Subtypes of type 4 cAMP phosphodiesterase: structure, regulation and selective inhibition. Trends Pharmacol. Sci. 17, 294-298.

Navaza, J., and Saludjian, P. (1997). AMoRe: an automated molecular replacement program package. Methods Enzymol. 276, 581-594. Otwinowski, Z., and Minor, W. (1997). Processing of X-ray diffraction data collected in oscillation mode. Methods Enzymol. 276, 307-326. Piaz, V.D., and Giovannoni, P. (2000). Phosphodiesterase 4 inhibitors, structurally unrelated to rolipram, as promising agents for the treatment of asthma and other pathologies. Eur. J. Med. Chem. 35, 463-480.

Reilly, M.P., and Mohler, E.R., III. (2001). Cilostazol: treatment of intermittent claudication. Ann. Pharmacother. 35, 48-56.

Richter, W., Unciuleac, L., Hermsdorf, T., Kronbach, T., and Dettmer, D. (2001). Identification of inhibitor binding of the cAMP-specific phosphodiesterase 4. Cell. Signal. 13, 287-297.

Rocque, W.J., Tian, G., Wiseman, J.S., Holmes, W.D., Zajac-Thompson, I., Willard, D.H., Patel, I.R., Wisely, G.B., Clay, W.C., Kadwell, S.H., et al. (1997). Human recombinant phosphodiesterase 4B2B binds (R)-rolipram at a single site with two affinities. Biochemistry 36, 14250-14261.

Rotella, D.P. (2002). Phosphodiesterase 5 inhibitors: current status and potential applications. Nat. Rev. Drug Discov. 1, 674-682.

Scott, A.I., Perini, A.F., Shering, P.A., and Whalley, I.L. (1991). In- 
patient major depression: is rolipram as effective as amitriptyline? Eur. J. Clin. Pharmacol. 40, 127-129.

Soderling, S.H., and Beavo, J.A. (2000). Regulation of cAMP and cGMP signaling: new phosphodiesterases and new functions. Curr. Opin. Cell Biol. 12, 174-179.

Souness, J.E., Aldous, D., and Sargent, C. (2000). Immunosuppressive and anti-inflammatory effects of cyclic AMP phophodiesterase (PDE) type 4 inhibitors. Immunopharmacology 47, 127-162.

Sturton, G., and Fitzgerald, M. (2002). Phosphodiesterase 4 inhibitors for the treatment of COPD. Chest 121, 192s-196s.

Thompson, W.J. (1991). Cyclic nucleotide phosphodiesterases: pharmacology, biochemistry and function. Pharmacol. Ther. 51, 13-33.

Torphy, T.J. (1998). Phosphodiesterase isozymes: molecular targets for novel antiasthma agents. Am. J. Respir. Crit. Care Med. 157, 351-370.

Torphy, T.J., Stadel, L.M., Burman, M., Cieslinski, L.B., Mclaughlin, M.M., White, J., and Livi, G.P. (1992). Coexpression of human cAMPspecific phosphodiesterase activity and high affinity rolipram binding in yeast. J. Biol. Chem. 267, 1798-1804.

Underwood, D.C., Kotzer, C.J., Bochnowicz, S., Osborn, R.R., Luttmann, M.A., Hay, D.W., and Torphy, T.J. (1994). Comparison of phosphodiesterase III, IV and dual III/IV inhibitors on bronchospasm and pulmonary eosinophil influx in guinea pigs. J. Pharmacol. Exp. Ther. 270, 250-259.

Xu, R.X., Hassell, A.M., Vanderwall, D., Lambert, M.H., Holmes, W.D., Luther, M.A., Rocque, W.J., Milburn, M.V., Zhao, Y., Ke, H., et al. (2000). Atomic structure of PDE4: insight into phosphodiesteras mechanism and specificity. Science 288, 1822-1825.

Zhao, A.Z., Yan, C., Sonnenburg, W.K., and Beavo, J.A. (1997). Recent advances in the study of $\mathrm{Ca} 2+/ \mathrm{Cam}$-activated phosphodiesterases. Signal Transduct. Health Dis. 31, 237-251.

\section{Accession Numbers}

The coordinates have been deposited in the Protein Data Bank under accession codes 10YM and 10YN. 